\title{
CORRESPONDENCE
}

resemble incomplete Kawasaki disease. This is considered to be secondary to immune dysregulation triggered after or in the later phase of the infection by severe acute respiratory syndrome coronavirus (SARS-CoV-2).
BHASKar SHENOY

Department of Pediatrics, Manipal Hospitals, Bangalore, Karnataka, India.

bshenoy@gmail.com

\section{Prehospital Management of Children with Dengue Fever Admitted in a Tertiary-Care Center}

Initial management of most children with dengue is done by local practitioners initially, and they are subsequently referred in the event of worsening of clinical condition. In the present study, we reviewed the initial management of dengue prior to being referred to our hospital, with special reference to the timing of the laboratory investigations, fluid management and use of platelet concentrates

A chart review of children referred as dengue to our hospital between September and November, 2019 was done after getting approval from the institutional ethics committee of our hospital. The data on pre-hospital management of these children recorded included: whether importance was given to packed cell volume (PCV) or platelet count; timing of dengue NS1 antigen testing, dengue $\operatorname{IgM}$ and $\operatorname{IgG}$ testing (card versus ELISA method); and treatment (fluid management, use of NSAIDs, antibiotics, and platelet transfusion). PCV is normally done at the time of presentation and repeated when clinically indicated (appearance of warning signs, progression to shock). When $\mathrm{PCV}$ is used for fluid titration, it is usually done once in 4 hours.

Among the 643 patients hospitalized for dengue during the study period, $129(20 \%)$ came by self-referral for fever because of their awareness regarding dengue. Most of the children who were brought by parents had dengue fever with no warning symptoms. $514(80 \%)$ were referred by physicians, of which a large proportion were not managed as per protocol [1]. Of the 514,385 children had dengue fever without warning symptoms, 103 children had dengue fever with warning symptoms, and 26 children had severe dengue.

Among the $80 \%$ of children who were referred from outside, in $20 \%$ of patients, PCV values were not given importance as fluids were not titrated based on PCV. On the other hand, in 30\% of patients, platelet counts were monitored thrice-a-day. Intravenous fluids were not given as per guidelines in $10 \%$ of patients, and they had received large volume of hypotonic fluids leading to signs of fluid overload, which was managed with fluid restriction and diuretics.
The timing of investigating NS1 antigen and $\operatorname{IgM}$ or IgG were not as per WHO guidelines [1], and card test was done in about $15 \%$ of patients. Despite there being no indication for platelet transfusion, $3 \%$ of patients had received platelet concentrates. Though the diagnosis of dengue was made, antibiotics were given in view of high spiking fever in $2 \%$ children. Mefenamic acid induced gastritis was seen in around $4 \%$ children. These children had normal PCV, no physical warning signs, and hence the vomiting was not considered as warning symptom and was attributed to mefenemic acid.

The children referred without warning symptoms needed just a day of observation while the ones with warning symptoms and severe dengue required four days of hospitalization. All the children in the study group improved and there was no mortality.

In a survey done in Singapore [2], where they tested the knowledge on diagnostic methods and clinical management of dengue using a questionnaire, there were significant issues in the understanding and diagnosis of dengue, particularly on the importance of using a diagnostic kit. There were also significant increase in awareness and practices of the best practices of dengue clinical management (choice of fluids and use of platelet concentrates) [2].

It is important to make sure that the protocols are uniformly followed by practitioners to ensure timely referral which in turn improves the outcome and reduces mortality. The present study underscores the gaps in knowledge about dengue management among practitioners, and we plan to conduct training activities for the same.

\section{Janani SankaR* AND Nithya Saravanan Kanchi Kamakoti Childs Trust Hospital Chennai, India. \\ *janani.sankar@yahoo.com}

\section{REFERENCES}

1. World Health Organization. National guidelines for clinical management of dengue fever. WHO Country Office for India, 2015. Available from https://apps.who.int/iris/handle/10665/ 208893

2. Ho TS, Huang MC, Wang SM, Hsu HC, Liu CC. Knowledge, attitude, and practice of dengue disease among healthcare professionals in southern Taiwan. J Formos Med Assoc. $2013 ; 112: 18-23$. 